\title{
Cascade Kinetics in an Enzyme-Loaded Aqueous Two-Phase System
}

\author{
Marko Pavlovic, Alexander Plucinski, Jianrui Zhang, Markus Antonietti, Lukas Zeininger,* \\ and Bernhard V. K. J. Schmidt*
}

Cite This: Langmuir 2020, 36, 1401-1408

Read Online

ACCESS | W W Metrics \& More | 国 Article Recommendations | st Supporting Information

ABSTRACT: Macromolecular crowding plays a critical role in the kinetics of enzymatic reactions. Dynamic compartmentalization of biological components in living cells due to liquid-liquid phase separation represents an important cell regulatory mechanism that can increase enzyme concentration locally and influence the diffusion of substrates. In the present study, we probed partitioning of two enzymes (horseradish-peroxidase and urate-oxidase) in a poly(ethylene glycol)-dextran aqueous two-phase system (ATPS) as a function of salt concentration and ion position in the Hofmeister series. Moreover, we investigated enzymatic cascade reactions and their kinetics within the ATPS, which revealed a strong influence of the ion hydration stemming from the background electrolyte on the partitioning coefficients of proteins

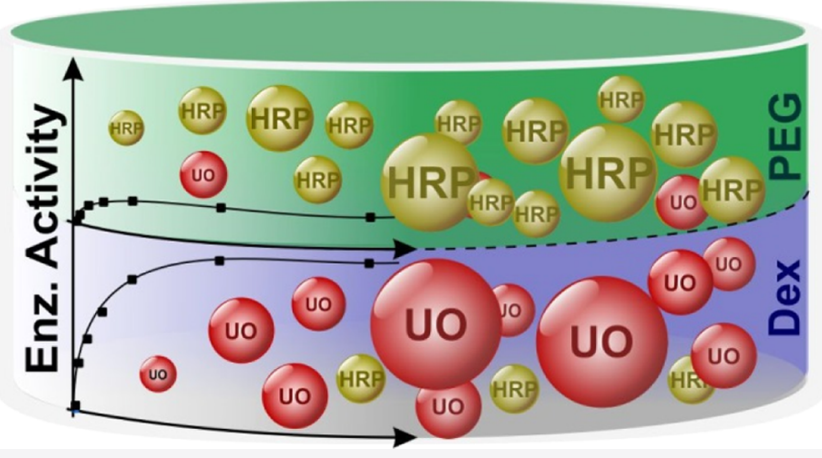
following the Hofmeister series. As a result, we were able to realize cross-partitioning of two enzymes because of different protein net charges at a chosen $\mathrm{pH}$. Our study reveals a strong dependency of the enzyme activity on the substrate type and crowding agent interaction on the final kinetics of enzymatic reactions in the ATPS and therefore provides substantial implications en route toward dynamic regulation of reactivity in synthetic protocells.

\section{INTRODUCTION}

The immense complexity of cell cytosol is responsible for a strong discrepancy between "in vitro" and "in vivo" experimental results on enzymatic reaction kinetics. Macromolecular crowding is one of the main reasons causing this discrepancy, ${ }^{1}$ as up to $40 \%$ of a cell volume can be occupied by crowding agents, with a concentration as high as $400 \mathrm{~g} / \mathrm{L}$. $^{2}$ Such a crowded environment has a significant influence on various reaction rates, ${ }^{3,4}$ protein conformation and aggregation, ${ }^{5}$ substrate diffusion, ${ }^{6}$ and water activity. ${ }^{7}$ As a result, reactions can be accelerated ${ }^{8-10}$ or impaired ${ }^{11}$ depending on the rate-limiting step in macromolecular crowded environments. If the reaction is diffusion-limited, an increasing crowding agent concentration results in a drop of diffusion and causes decreased reaction rates. In contrast, if the ratedetermining step is the substrate conversion to the transition (dimer) state, reaction rates can be increased by promotion of the substrate association. An additional phenomenon arising from macromolecular crowding is molecular confinement. Often caused by either cytoskeleton or numerous chaperonin proteins, this effect is related to the presence of the macromolecules in small compartments inside a cell. Such confined space can successfully promote protein folding and prevent escaping of encapsulated polypeptide. ${ }^{12}$ Moreover, a high macromolecular content alters the properties of water by interfering with hydrogen bonding ${ }^{13}$ or leaving less solvent molecules for the substrate and the enzyme that can further cause changes in the enzyme kinetics.

More recently, phase separation inside the cell has attracted the focus of scientists, which is another important phenomenon representing a consequence of elevated fractions of crowding agents. ${ }^{14,15}$ Demixing is the main requirement for the formation of the numerous membrane-less organelles ( $P$ bodies, stress granules, Cajal bodies, nucleoli, etc.). ${ }^{16-18}$ In this way, cells ensure locally high concentrations of substrates and/or enzymes, while the absence of membranes allows rapid mass transfer across the permeable phase boundary at the same time, thus maximizing diffusion. In addition, as a result of only minor external stimuli, these organelles rapidly mix, rendering them extremely dynamic. For instance, it has been recently reported that phase transition is the main trigger for the development of some diseases such as amyotrophic lateral sclerosis. ${ }^{19}$ While it took some time for biology to link this phenomenon to several aspects of cell behavior, chemists were using phase separation in a solely aqueous environment as a tool in separation chemistry frequently, especially via polymer demixing. $^{20-25}$ This similarity to living cells represents a basis

Received: January 21, 2020

Published: January 24, 2020 
for an efficient way to separate proteins, either from other proteins or from debris, while keeping their native structure intact. As such, the effect of phase separation in an aqueous two-phase system (ATPS) has been utilized for the purification of proteins and nanoparticles, ${ }^{26,27}$ as well as for the generation of water-in-water emulsions using Pickering stabilizers. ${ }^{28-30}$

Dispersed ATPS is particularly useful for enzymatic catalysis because of the existence of fully permeable boundaries together with selective compartmentalization..$^{31-37}$ To this end, a sophisticated control of multienzyme cascades requires control over the enzyme partitioning in individual phases. Tuning ATPS by varying $\mathrm{pH}$, adding simple salts, and changing polymer molar masses or volume ratios can impart crucial differences between the phases in order to separate enzymes. At the same time, low interfacial tension and high water content allow maintainance of enzyme activity and their native state. $^{38}$ Owing to electrochemical, hydrophobic, biospecific, size, and conformational contributions, ${ }^{24}$ the partitioning coefficients of proteins in the ATPS can be influenced by the polymer type and composition. In this context, enzymatic activity in crowded milieu has been studied for the purpose of separation. ${ }^{39}$ While the majority reports an increase in the Michaelis-Menten constant $\left(K_{\mathrm{m}}\right)$ by addition of crowding agents, others suggest an amplified affinity of the substrates toward enzymes. ${ }^{40}$ Although a tuning of enzymatic reactivity is conventionally described as a function of their conformation in crowded microenvironments, the additional effect of limited substrate diffusion poses a challenge in predicting the kinetics of enzymatic reactivity and requires optimization toward dynamic regulation of enzymatic reactivity and the design of consistent and reproducible cascade reactions.

In the present study, we investigated the kinetics of an enzymatic cascade reaction inside an enzyme-loaded ATPS as a function of the substrate type and crowding agent interaction (Scheme 1). Specifically, we found that horseradish-peroxidase (HRP) partitioning critically depends on the type of background electrolyte, and the entire trend can be predicted via the position of the respective ions in the Hofmeister series. En route toward a dynamic regulation of enzymatic reactivity in crowded environments, we started with an optimization of the cross-partitioning of enzymatic constituents in different ATPSs with variations in ionic strength and the type of added salt, followed by investigations of the effect crowded milieu has not only on the enzyme's native conformation but also on the translational diffusion of several substrates. In addition, insights on the cross-partitioning of HRP and urate-oxidase (UO) in phase-separated aqueous mixtures of salts and polymers were combined with investigations of the translational diffusion of different HRP substrates [guaiacol and 2,2'-azino-bis(3ethylbenzothiazoline-6-sulfonic acid) (ABTS) diammonium salt]. A subsequent study of the kinetics of cascade reactions in the phase-separated aqueous mixtures revealed a strong influence of the substrate-polymer interactions on the diffusion rates and enzyme kinetics, while the native structure of the enzymes remained unaffected, which provides substantial implications for both, a better understanding of the enzymatic reactions inside living cells and for the realization of artificial systems capable of dynamically regulating enzymatic reactivity.

\section{EXPERIMENTAL SECTION}

Materials. HRP (type VI, EC number 1.11.1.7) and UO (EC number 1.7.3.3) were purchased in the form of lyophilized powder
Scheme 1. Schematic Outline of the Experimental Procedure for Investigating the Kinetics of Enzymatic Cascade Reactions Inside Phase-Separated Polymer Mixtures Starting from Unstable Poly(ethylene glycol) (PEG)-Dextran (Dex) Emulsion through Phase Separation and Enzyme Partitioning Until Enzymatic Activity Measurements (HRP: Horseradish-Peroxidase; UO: Urate Oxidase)



from Sigma-Aldrich and used without any further purification. ABTS diammonium salt, deuterium oxide, ethanol, guaiacol, PEG of different molar masses, phosphoric acid, and various simple salts were obtained by the same supplier. Coomassie Brilliant Blue G-250 (98\%) dye for Bradford assay was obtained from Thermo Fisher. Dex polymers of $500 \mathrm{k}$ and $40 \mathrm{k}$ were purchased from TCI Deutschland $\mathrm{GmbH}$. Phosphate buffer $0.2 \mathrm{M}$ and $\mathrm{pH} 8$ was obtained from Alfa Aesar.

Partitioning Coefficient. Partitioning coefficients $(K)$ were determined by comparing the enzyme concentration in the top and bottom phases (eq 1) by performing the Bradford test, as described previously. ${ }^{41,42}$

$$
K=\frac{C_{\text {top }}}{C_{\text {bottom }}}
$$

$C_{\text {top }}$ and $C_{\text {bottom }}$ represent the enzyme concentration in the top and bottom phases. In brief, Bradford reagents were prepared by dissolving Coomassie Brilliant Blue G-250 dye in $50 \mathrm{~mL}$ of $95 \%$ ethanol. Dye solution was prepared with $100 \mathrm{~mL}$ of $85 \%$ phosphoric acid and $850 \mathrm{~mL}$ of Milli-Q water. Initially, stock solutions of 1000 $\mathrm{mg} / \mathrm{L}$ HRP and UO were prepared, and two calibration curves were plotted in the range from 1 to $20 \mathrm{mg} / \mathrm{L}$. Absorption spectra were recorded with a T70+ UV/vis spectrometer (PG Instruments Ltd). Two characteristic absorption bands, at 465 and $595 \mathrm{~nm}$, originate from the free dye and dye-enzyme complex, respectively. Difference between these absorptions was determined and plotted as a function of the enzyme concentration, resulting in the calibration curves. Local enzyme concentrations were determined by diluting $100 \mu \mathrm{L}$ of the tested phase with $100 \mu \mathrm{L}$ of Milli-Q water and $800 \mu \mathrm{L}$ of the Bradford reagent. The partitioning coefficient was then quantified by linearly 
fitting the measured absorbance ratios and comparing them to the calibration curve.

Enzymatic Assay. Determination of the cascade reaction kinetics was performed in a slightly modified manner in comparison to the standard guaiacol and ABTS assay for peroxidase activity. Specifically, $\mathrm{H}_{2} \mathrm{O}_{2}$ was not added as a separate reagent but produced by the first enzymatic reaction of oxidation of uric acid to 5-hydroxyisourate catalyzed by $\mathrm{UO}$ as a side product. For this purpose, $0.2 \mathrm{U}$ of both enzymes were mixed with $0.03 \mathrm{~mm}$ uric acid, $5 \mathrm{~mm}$ phosphate buffer, and guaiacol concentration that was varied from 0.5 to $50 \mathrm{~mm}$ inside the spectrophotometry cell. Initial increase in absorbance at $470 \mathrm{~nm}$ was monitored as a function of time, and the results were analyzed using the Michaelis-Menten model (eq 2) ${ }^{43}$

$$
v=\frac{v_{\max }[\mathrm{S}]}{K_{\mathrm{m}}+[\mathrm{S}]}
$$

in which $v$ stands for the rate of the reaction, $[\mathrm{S}]$ for the substrate concentration, and $K_{\mathrm{m}}$ and $v_{\max }$ refer to Michaelis-Menten constant and maximum rate of the reaction, respectively. In the case of a cascade reaction, inhibition by polymers, guaiacol, and ABTS concentration in separate assays was set to $25 \mathrm{~mm}$ or $1 \mathrm{~mm}$, respectively, and $\mathrm{w} / \mathrm{v} \%$ of either PEG or Dex was set to be between 3 and 15 . The initial rate of the formation of the colored product was recorded at $470 \mathrm{~nm}$ for guaiacol, or at $405 \mathrm{~nm}$ for $\mathrm{ABTS}$, and plotted as a function of polymer concentration.

Circular Dichroism. Circular dichroism (CD) spectra were recorded using a $2 \mathrm{~mm}$ path length quartz cuvette and an Applied Photonics Chirascan qCD spectrometer. Samples were prepared by dissolving $10 \mathrm{mg} / \mathrm{mL}$ of both enzymes separately, in the absence or presence of $10 \mathrm{w} / \mathrm{v} \%$ of either PEG or Dex. Entire spectra were recorded in the far UV range $(200-250 \mathrm{~nm})$.

Diffusion-Ordered Spectroscopy-NMR. In order to compare the translational diffusion coefficients of substrate molecules in buffer to polymer crowded solutions, diffusion-ordered NMR (DOSYNMR) spectroscopy was employed. A $100 \mathrm{~mm}$ solution of substrate (guaiacol or ABTS) was prepared in a total volume of $600 \mu \mathrm{L}$ with $\mathrm{D}_{2} \mathrm{O}$ as solvent. This solution was used as prepared or enriched by either $60 \mathrm{mg}$ of PEG or Dex, which corresponds to $10 \mathrm{w} / \mathrm{v} \%$ of polymer in actual samples. DOSY-NMR was performed at $600 \mathrm{MHz}$ (Agilent 600 premium shielded) with the Dbppste_CC pulse sequence, and the obtained data is presented in the diffusion ordered representation.

\section{RESULTS AND DISCUSSION}

Tuning the ATPS for Cross-Partitioning. With the purpose to investigate enzymatic cascade reactions in environments with high crowding agent concentration, we started with an investigation of the cross-partitioning of two enzymes in a phase-separated aqueous polymer mixture comprising a PEGrich upper and Dex-rich lower phase. For the cascade reaction scheme, we opted for enzymatic oxidation of uric acid by UO, which produced peroxide that was subsequently converted in a peroxidase-mediated oxidation of two different substrates, namely, guaiacol and ABTS. To investigate the crosspartitioning and enzyme activity of UO and HRP in aqueous polymer mixtures of neutral $\mathrm{pH}$, the entire cascade reaction was performed in the individual phase-separated compartments to facilitate spectrometrical monitoring of the reaction progress in a transparent medium.

Both enzymes display relatively low molar masses of 33.4 and $44.5 \mathrm{kDa}$, respectively. One critical distinction between them is the isoelectric point (IP) of these enzymes, and therefore, their charge at neutral $\mathrm{pH}$. UO has an IP of 5, while the IP of HRP utilized in this study is 9. As a consequence, at $\mathrm{pH} \mathrm{7,} \mathrm{UO} \mathrm{is} \mathrm{predominantly} \mathrm{negatively} \mathrm{charged,} \mathrm{while} \mathrm{at} \mathrm{the}$ same time, the net charge of HRP was positive. This fact enabled an efficient cross-partitioning of these catalysts. Many previous studies employed the polymer-salt ATPS for partitioning because of very good partitioning coefficients $(K)$. Nevertheless, enzymes and proteins, in general, tend to have strong partitioning to the salt-rich phase, which is beneficial for purification processes but not for crosspartitioning. In the present case, the polymer-salt ATPS gave strong enrichment of the salt-phase with both enzymes, which has been reported earlier for HRP. ${ }^{44}$ In contrast, partitioning coefficients can be tuned by varying molar mass of the polymers in the polymer-polymer ATPS. In order to achieve higher $K$ values of HRP, it is necessary to use lower molar mass PEGs and higher molar mass Dex. In our study, 3k PEG and 500k Dex provided a good starting point for further tailoring of the system that could eventually be used in the cascade reaction (Figure $\mathrm{S} 1 \mathrm{~b}$ ). With the aim to achieve best possible partitioning and to investigate the effect of the polymers on enzyme kinetics, we chose comparatively high polymer concentration of $10 \mathrm{w} / \mathrm{v} \% \mathrm{PEG}$ and $15 \mathrm{w} / \mathrm{v} \% \mathrm{Dex}$ (Figure S2). This total polymer concentration of $25 \mathrm{w} / \mathrm{v} \%$ is well above the binodal and guaranteed high compositional diversity among top and bottom phases. ${ }^{45}$ The influence of polymer molar masses on HRP $K$ values in PEG-Dex ATPS is summarized in the inset of Figure S2 in the Supporting Information. It is clear that enzyme preference to one or the other phase can be adjusted simply by changing polymer molar mass. While in the system with low PEG and high Dex molar mass (system used in the proceeding parts of the study) that granted $K$ values of HRP higher than 1 , HRP preferred a lower Dex phase in the case of low Dex and high PEG molar mass.

Upon optimization of the polymer system for efficient crosspartitioning of enzymes, we investigated the influence of buffer concentration, together with background salt. As displayed in Figure 1 a, varying $\mathrm{NaCl}$ concentration while maintaining buffer at a fixed concentration can induce serious changes in partitioning coefficients, namely, best cross-partitioning, or highest difference of $K$ of HRP and UO was achieved at salt concentrations around $50 \mathrm{~mm}$. Any further salt addition "pushed" UO to the top, that is, the PEG-rich phase, while similar phenomena occurred also at lower salt levels. In contrast, partitioning of HRP was not significantly affected by these variations. At the same time, the importance of buffer concentration on partitioning was observed. While HRP partitioning was not affected, elevated phosphate buffer concentrations notably decreased the efficiency by increasing the $K$ value of UO. The difference in the appearance of the partitioning graph in phosphate or $\mathrm{NaCl}$ buffer can be attributed to variations in ionic strength. Overall, phosphate and $\mathrm{NaCl}$ buffer showed a rather opposite partitioning trend for HRP, which can be related to Hofmeister series. Namely, $\mathrm{pH} 7.8$ phosphate buffer contains sodium cations that counter negatively charged hydrogenphosphate and dihydrogenphosphate anions. Both of these anions are highly hydrated and are placed at the opposite side of Hofmeister series in comparison to chloride. This is the main reason why increasing $\mathrm{NaCl}$ concentration promotes desired protein partitioning, while lowering phosphate buffer concentration. Therefore, further investigations regarding the correlation of Hofmeister series with enzyme partitioning were performed. According to our results, the ATPS composed of $10 \mathrm{w} / \mathrm{v} \%$ PEG $3 \mathrm{k}$ and $15 \mathrm{w} / \mathrm{v}$ $\%$ Dex $500 \mathrm{k}$ with the $50 \mathrm{~mm}$ background salt concentration and $\mathrm{pH} 7.8$ set by $5 \mathrm{~mm}$ phosphate buffer was identified as an ideal environment for an investigation of the cascade reaction. 

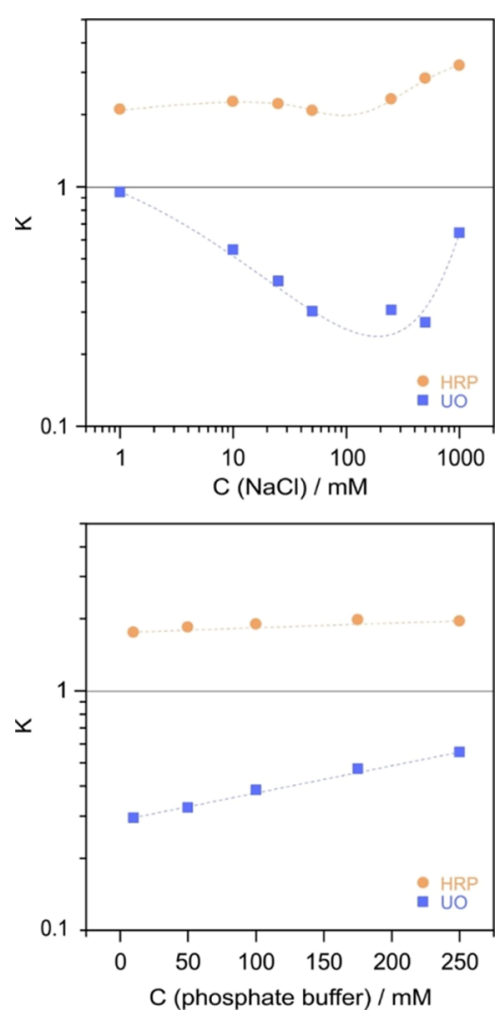

Figure 1. Partitioning coefficient $(K)$ of HRP (orange dots) and UO (blue squares) in the presence of various $\mathrm{NaCl}$ (a) or phosphate buffer (b) concentrations.

Hofmeister Series. Next, the cross-partitioning of positively charged HRP and negatively charged $\mathrm{UO}$ in the PEG-Dex ATPS was followed at $\mathrm{pH} 7.8$ in the presence of different salts (Figure 2). By using sodium salts with different anions or chloride salts with various cations, both anionic and cationic Hofmeister series were probed. In addition, we tried to extend the classical series with a 1-butyl-3-methylimidazolium $\left(\mathrm{BMIM}^{+}\right)$cation, a common constituent of numerous ionic liquids. $^{46-49}$ All measurements were performed at three different salt concentrations varying from 1 to $50 \mathrm{~mm}$.

As displayed in Figure 2, efficiency of the cross-partitioning was comparable for all ions at low concentration; however, by increasing the concentration, the hydrophobicity of the ions (position in Hofmeister series) started to influence the crosspartitioning. While positively charged HRP tended to be less sensitive to the type of the salt and salt concentration, UO partitioning coefficient strongly depended on both factors. We observed a significant increase of UO partitioning coefficient starting from 0.2 for $\mathrm{SCN}^{-}$up to 0.7 for the most hydrophilic citrate anion. The dashed line connecting $50 \mathrm{~mm}$ case for two enzymes of the same salt type (resolution), show-cases a notable drop by decreasing anion hydrophobicity. Most hydrophobic anions generated highest cross-partitioning, whereas opposite effects were observed for cations (Figure 2b). More hydrophobic cations caused both enzymes to distribute equally in the phases with $K$ values close to 1 , while the most hydrophilic $\mathrm{Li}^{+}$proved to be most effective for separating these enzymes in two phases. The latter effect was more pronounced for UO than in the case of HRP. The importance of the presence of the background electrolyte type was attributed to the partitioning of the ions themselves. More hydrophobic ions tend to prefer the less hydrophilic PEG-rich

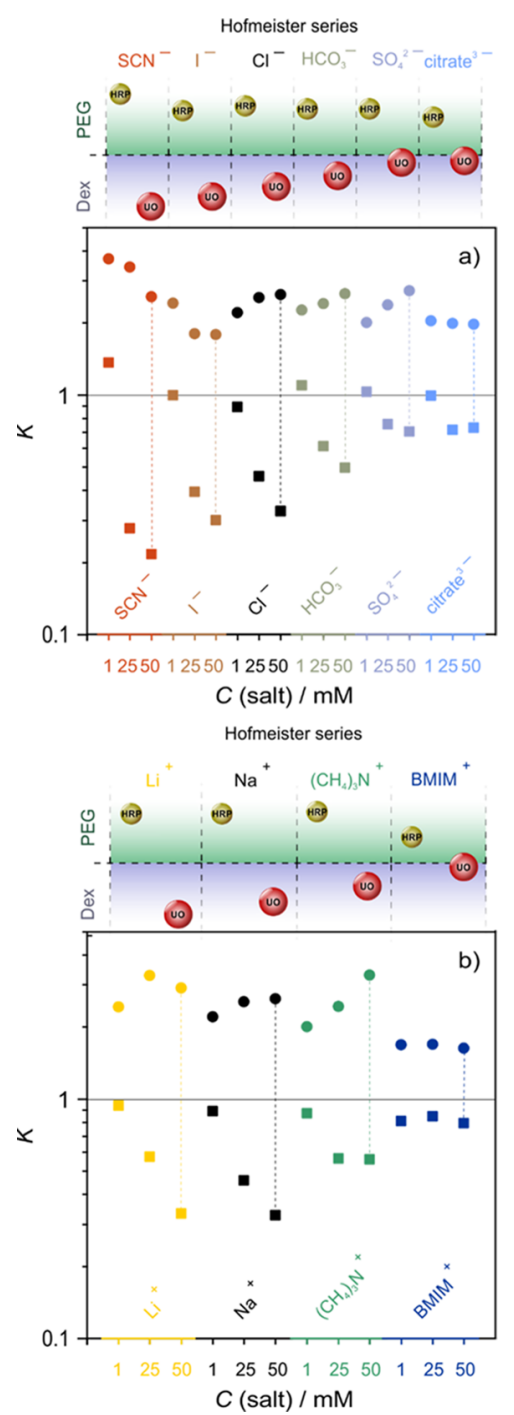

Figure 2. Partitioning coefficient $(K)$ of HRP (circles) and UO (squares) in the presence of various anion (a) and cation (b) concentrations. The $\mathrm{Na}^{+}$cation was used as a counter-ion for each anion, while $\mathrm{Cl}^{-}$was utilized as the anion for investigation of the cation effect. All experiments were performed at $\mathrm{pH} 7.8$ and $5 \mathrm{~mm}$ phosphate buffer concentration.

phase (water-poor phase in comparison to the water-rich Dex phase) and therefore induced a more negative Donnan potential between the two phases. ${ }^{20,50,51}$

Enzymatic Activity in the ATPS. To leverage the Donnan potential in a beneficial way, we chose a $\mathrm{pH}$ at which HRP and UO have opposite net charges. In such systems, two enzymes tend to partition in the opposite phases. Adverse effects in the case of very hydrophilic negatively charged anions, such as citrate, are attributed to a preferred partitioning of the anion in the Dex-rich phase that can induce undesired Donnan potential in our system. The same phenomenon occurred for cations, however, this time using more hydrophilic cations, such as $\mathrm{Li}^{+}$, that prefer the Dex phase, with higher water content, and caused the desired Donnan effect as well as promoted partitioning of HRP in the upper phase and UO in the lower phase. Consequently, using more hydrophilic cations and more hydrophobic anions resulted in a negative Donnan potential that showed, together with different chemical compositions of the phases, significant electrochemical 
Scheme 2. Two Cascade Reactions Investigated in the Current Study ${ }^{a}$

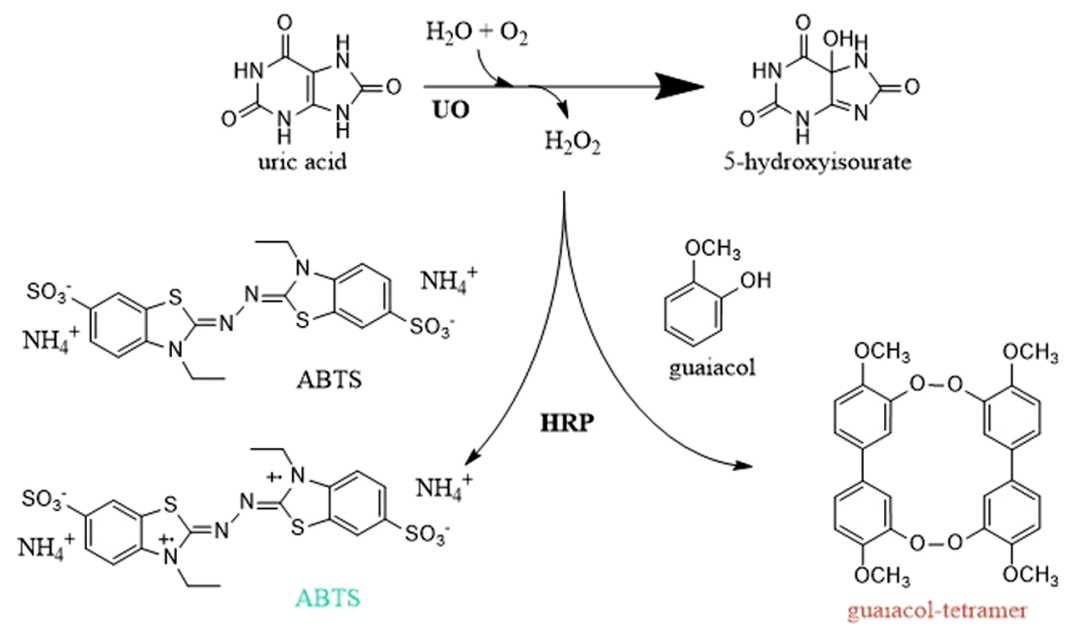

${ }^{a}$ Both of them starting from the oxidation of uric acid, which is followed by subsequent formation of the colored product from either ABTS or guaiacol oxidation.

potential for the most efficient cross-partitioning of the two enzymes. It should be noted that we chose $\mathrm{NaCl}$ at $50 \mathrm{~mm}$ as a background electrolyte for all further investigations as a compromise between appropriate concentrations to cause significant partitioning, while suppressing interference with the native protein structure.

In order to probe enzymatic activity of the HRP-UO enzyme cascade (Scheme 2), an ATPS composed of $10 \mathrm{w} / \mathrm{v} \%$

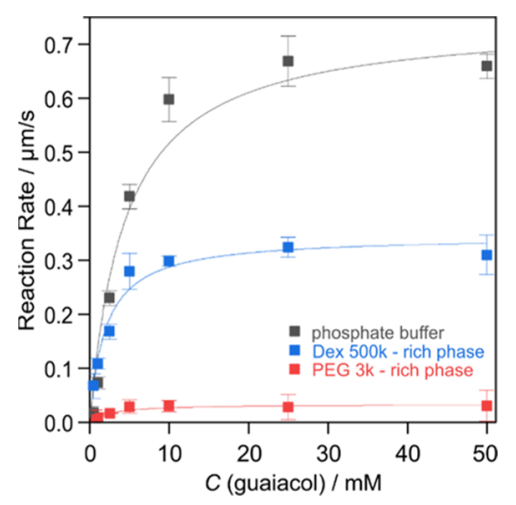

Figure 3. Enzyme cascade kinetics performed in Milli-Q water (grey squares), Dex-rich bottom phase (blue squares), and PEG-rich top phase (red squares). Two phases were obtained after equilibration overnight. All experiments were done at $\mathrm{pH} 7.8$ set by $5 \mathrm{~mm}$ phosphate buffer and $50 \mathrm{~mm} \mathrm{NaCl}$ as a background electrolyte.

PEG 3k and 15 w/v \% Dex 500k, $50 \mathrm{~mm} \mathrm{NaCl}$ concentration, and $\mathrm{pH} 7.8$ set by $5 \mathrm{~mm}$ phosphate buffer was prepared. In addition, $0.2 \mathrm{U} / \mathrm{mL}$ (where $\mathrm{U}$, enzymatic unit, stands for an amount of enzyme that catalyzes conversion of $1 \mu \mathrm{mol}$ of the substrate per minute) of both UO $(100 \mathrm{mg} / \mathrm{mL})$ and HRP (5 $\mathrm{mg} / \mathrm{mL}$ ) was added, and the entire system was allowed to equilibrate overnight. Subsequently, the ability of the PEG-rich and Dex-rich phases as environments for the cascade reaction (oxidation of uric acid followed by an oxidation of the dye i.e., detected spectrophotometrically) was tested and compared to the case of the absence of polymer (Figure 3).

In this case, partitioning of the enzymes took place according to the $K$ values from Figure 1. Accordingly, by

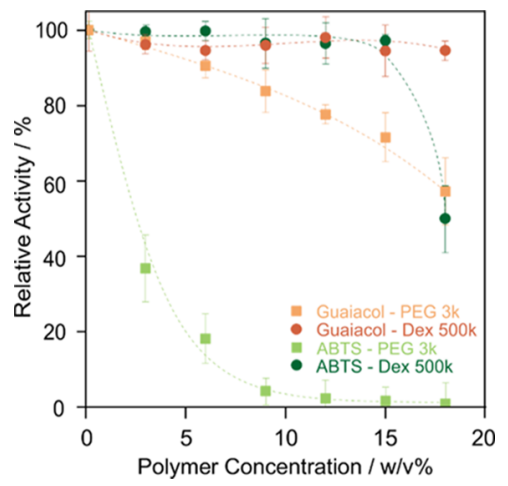

Figure 4. Relative activity of the enzyme cascade, measured by absorbance of guaiacol or the ABTS oxidation product in the presence of either PEG 3k (squares) or Dex 500k (circles). All experiments were performed at $\mathrm{pH} 7.8$ set by $5 \mathrm{~mm}$ phosphate buffer and $50 \mathrm{~mm}$ $\mathrm{NaCl}$ as a background electrolyte.

addition of $0.2 \mathrm{U} / \mathrm{mL}$ of both UO and HRP to our ATPS, the PEG-rich phase absorbs approximately $0.264 \mathrm{U} / \mathrm{mL}$ of HRP and $0.066 \mathrm{U} / \mathrm{mL}$ of $\mathrm{UO}$, while in the Dex-rich phase, $0.132 \mathrm{U} /$ $\mathrm{mL}$ of HRP and $0.333 \mathrm{U} / \mathrm{mL}$ of $\mathrm{UO}$ are present. This is a complex system with an unforeseeable activity of both phases; however, because of the significantly denser Dex-rich phase, we expected to observe lower activities in that phase solely because of the hydrodynamic effect. Nevertheless, we observed opposite results. Both PEG and Dex phases exhibited lower $V_{\max }$ (maximum rate of the reaction) values of 0.03 and 0.34 $\mu \mathrm{mol} / \mathrm{s}$, respectively, in comparison to $0.75 \mu \mathrm{mol} / \mathrm{s}$ for the phosphate buffer system. The main cause of the overall lower activity behavior was attributed to partitioning, which, however, did not explain the high enzymatic activity of the lower Dex-phase and barely measureable activity of the upper PEG-phase. The values regarding the Michaelis-Menten fit are collated in Table S1. It is evident that the upper ATPS phase has one order of magnitude lower $V_{\max }$ although, all $K_{\mathrm{m}}$ are similar. Thus, the observed behavior could be explained by the ability of the PEG polymer to interfere with hydrophobic interactions inside the protein structure and partially denature enzymes, as previously reported for some specific interaction or crowding agents with substrates. ${ }^{5,52}$ 



Figure 5. CD spectra of HRP (a) and UO (b) in the absence of any polymer (black squares) and in the presence of PEG 3k (red triangles) or Dex 500k (green dots).

In pursuance of understanding the previously described discrepancies in enzymatic activity of the two phases, we performed several probes investigating reaction rates of cascade reaction in pure PEG or Dex solution at different concentrations without a formation of the ATPS but with the same ionic strength and $\mathrm{pH}$ values as in ATPS assays. For this purpose, $0.2 \mathrm{U}$ of both enzymes were added to the reaction mixture together with $0.03 \mathrm{~mm}$ of uric acid, $50 \mathrm{~mm}$ of guaiacol, or $1 \mathrm{~mm}$ ABTS and varying concentrations of either PEG or Dex. Initial rates of this reaction were normalized by the rate of the reaction without any polymer and plotted versus the polymer concentration (Figure 4). As a result, even elevated Dex concentration did not alter the rate of the reaction significantly for both substrates (with an exception of $18 \mathrm{w} / \mathrm{v} \%$ for ABTS as a substrate). In contrast, already $10 \mathrm{w} / \mathrm{v} \%$ of the PEG rate of the cascade reaction dropped close to $80 \%$ for guaiacol and almost to $0 \%$ for ABTS. Alongside with our findings that PEG lowers the rate of the reaction, the different degree of inhibition depending on the substrate type remained to be studied.

CD Spectroscopy of Enzymes in Different Media. CD spectroscopy proved to be a useful tool to investigate any conformational changes in the protein secondary structure. Namely, CD spectra of both enzymes were recorded in the range from 200 to $250 \mathrm{~nm}$ in phosphate buffer and in the presence of $10 \mathrm{w} / \mathrm{v} \%$ of either PEG or Dex (Figure 5). Both enzyme spectra exhibited characteristic maxima at 208 and 220 $\mathrm{nm}$, typical for proteins with a high content of an $\alpha$-helical structure. Upon addition of $10 \mathrm{w} / \mathrm{v} \%$ of any of the two polymers, no significant shift of the peaks was observed. Signal intensity was also maintained providing a proof of a preserved enzyme secondary helical structure upon polymer addition. Thus, the variations in activity of the enzymes in the ATPS did not stem from the enzyme denaturation or any interaction between polymers and enzymes but can be solely attributed to the interaction between substrates and polymers.

Substrate Diffusion Coefficient in Different Media. Therefore, we next set out to investigate the substrate mobility in the respective medium via measurements of diffusion. In order to measure the translational diffusion of substrates in the absence and presence of polymers, we employed DOSY-NMR. Higher concentrations of the polymer showed to significantly influence the measurement and simply screen the peaks that originate from the substrate molecules. In addition, this PEG dose was enough to induce 15 and $90 \%$ drop of activity for guaiacol and ABTS as the substrates, respectively. The obtained results are displayed in Figure 6, where a distribution of the diffusion coefficients $(D)$ is plotted against characteristic chemical shifts. For the better overview of the results, diffusion coefficients are given in Table S2 together with a percentage of the translational diffusion decrease. We expected a higher decrease of the diffusion for the more viscous Dex media as it is projected by the Stokes-Einstein equation. In spite of that, diffusion coefficient is not always proportional to viscosity because of possible short-range interaction that occurs. ${ }^{53}$ In this scenario, diffusion is governed partially by media viscosity but majorly by specific short-range interaction. ${ }^{54}$

As revealed by our studies, a drop in diffusion in Dex media of 30 and $39 \%$ for guaiacol and ABTS, respectively, could be explained by the increased viscosity of the system. Nevertheless, these values are not comparable to 44 and 52\% drop of the same compounds in media that are composed of PEG as a crowding agent, which provided for a strong indication of specific attractive forces between both guaiacol-PEG and ABTS-PEG that influence the diffusion. A small shift in the proton signal from 0.95 to 1.09 was attributed to a stronger
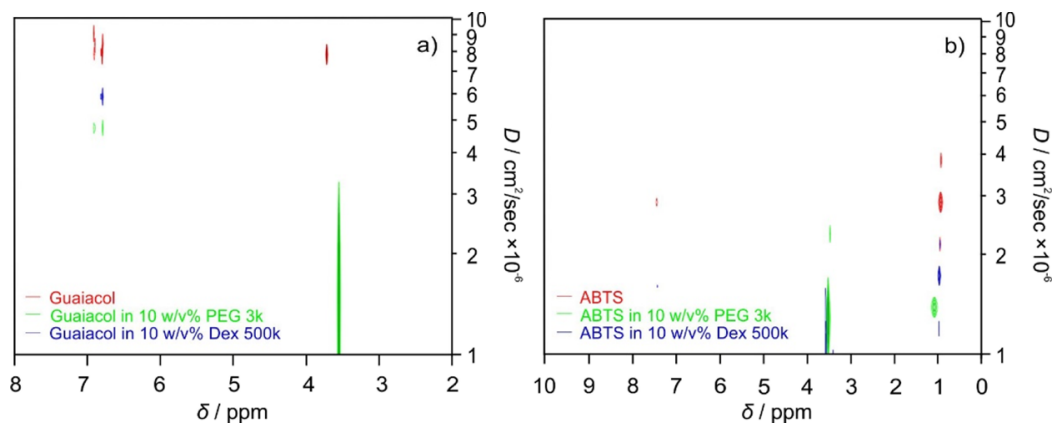

Figure 6. DOSY-NMR of guaiacol (a) and ABTS (b) in the absence of any polymers (red signal) and in the presence of $10 \mathrm{w} / \mathrm{v} \%$ of PEG (green signals) or Dex (blue signals). 
ABTS-PEG interaction that can potentially cause a decrease in diffusion coefficient, and subsequently, in reduced ability of HRP to oxidize this substrate. A similar shift has not been observed in guaiacol-PEG mixture. Taken together, interactions between PEG and both substrates can decrease the rate of enzymatic reaction significantly. Similar interactions are not present among complex-branched polysaccharides, such as Dex, and substrates, which ensured high enzyme catalyzed reaction rates.

\section{CONCLUSIONS}

In conclusion, the partitioning of the two enzymes, HRP and UO, was investigated in different ATPSs with variations in ionic strength and the type of added salt. High molar mass Dex and low molar mass PEG were chosen as ATPS constituents because of the most efficient cross-partitioning of previously mentioned cascade enzymes. The effect of the ion hydrophobicity on partitioning of proteins is described in terms of the Hofmeister series. Moreover, kinetics of cascade reactions in crowded environments were investigated. Discrepancies in reaction rates between the PEG-rich and Dex-rich phase were observed that could not be explained by changes in native enzyme confirmation as proven proved by $\mathrm{CD}$ measurements. Because the difference in activity was dependent on the type of the utilized HRP substrate, this phenomenon was correlated to different translational diffusion coefficients of the different substrates (guaiacol and ABTS) in solutions of high polymer concentration. The associated understanding of the enzyme partitioning and influences on the kinetics of enzymatic reactions in the crowded ATPS will serve as an important tool for separation chemistry and has several implications for understanding and dynamically regulating enzymatic reactivity in macromolecular crowded environments such as cells.

\section{ASSOCIATED CONTENT}

\section{(5) Supporting Information}

The Supporting Information is available free of charge at https://pubs.acs.org/doi/10.1021/acs.langmuir.0c00186.

Additional partition measurement results, kinetic parameters, and diffusion coefficients (PDF)

\section{AUTHOR INFORMATION}

\section{Corresponding Authors}

Lukas Zeininger - Department of Colloid Chemistry, Max Planck Institute of Colloids and Interfaces, 14476 Potsdam, Germany; Email: lukas.zeininger@mpikg.mpg.de

Bernhard V. K. J. Schmidt - Department of Colloid Chemistry, Max Planck Institute of Colloids and Interfaces, 14476 Potsdam, Germany; School of Chemistry, University of Glasgow, G128QQ Glasgow, U.K.; $\odot$ orcid.org/0000-0002-35807053; Email: bernhard.schmidt@glasgow.ac.uk

\section{Authors}

Marko Pavlovic - Department of Colloid Chemistry, Max Planck Institute of Colloids and Interfaces, 14476 Potsdam, Germany

Alexander Plucinski - Department of Colloid Chemistry, Max Planck Institute of Colloids and Interfaces, 14476 Potsdam, Germany; School of Chemistry, University of Glasgow, G128QQ Glasgow, U.K.

Jianrui Zhang - Department of Colloid Chemistry, Max Planck Institute of Colloids and Interfaces, 14476 Potsdam, Germany
Markus Antonietti - Department of Colloid Chemistry, Max Planck Institute of Colloids and Interfaces, 14476 Potsdam,

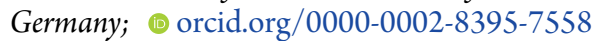

Complete contact information is available at: https://pubs.acs.org/10.1021/acs.langmuir.0c00186

\section{Author Contributions}

The manuscript was written through contributions of all authors. All authors have given approval to the final version of the manuscript.

Notes

The authors declare no competing financial interest.

\section{ACKNOWLEDGMENTS}

The authors are grateful for funding from the Max-Planck society. M.P. acknowledges financial support from the Swiss National Science Foundation with the project number P2GEP2 181528. B.S. and A.P. acknowledge funding from the University of Glasgow and the German Research Foundation (grant no. SCHM 3282/3-1). J.Z. acknowledges funding from the China Scholarship Council. L.Z. is grateful for funding through the Emmy-Noether program of the German Research Foundation (grant no. ZE 1121/3-1).

\section{REFERENCES}

(1) Ellis, R. J. Macromolecular crowding: an important but neglected aspect of the intracellular environment. Curr. Opin. Struct. Biol. 2001, $11,114-119$.

(2) Ellis, R. J.; Minton, A. P. Join the crowd. Nature 2003, 425, 2728.

(3) Kim, J. S.; Yethiraj, A. Effect of macromolecular crowding on reaction rates: a computational and theoretical study. Biophys. J. 2009, 96, 1333-1340.

(4) Minton, A. P. The influence of macromolecular crowding and macromolecular confinement on biochemical reactions in physiological media. J. Biol. Chem. 2001, 276, 10577-10580.

(5) Kuznetsova, I.; Turoverov, K.; Uversky, V. What macromolecular crowding can do to a protein. Int. J. Mol. Sci. 2014, 15, 23090-23140.

(6) Kekenes-Huskey, P. M.; Scott, C. E.; Atalay, S. Quantifying the Influence of the Crowded Cytoplasm on Small Molecule Diffusion. J. Phys. Chem. B 2016, 120, 8696-8706.

(7) Derham, B. K.; Harding, J. J. The effect of the presence of globular proteins and elongated polymers on enzyme activity. Biochim. Biophys. Acta, Proteins Proteomics 2006, 1764, 1000-1006.

(8) Totani, K.; Ihara, Y.; Matsuo, I.; Ito, Y. Effects of Macromolecular Crowding on Glycoprotein Processing Enzymes. J. Am. Chem. Soc. 2008, 130, 2101-2107.

(9) Norris, M. G. S.; Malys, N. What is the true enzyme kinetics in the biological system? An investigation of macromolecular crowding effect upon enzyme kinetics of glucose-6-phosphate dehydrogenase. Biochem. Biophys. Res. Commun. 2011, 405, 388-392.

(10) Paudel, B. P.; Fiorini, E.; Börner, R.; Sigel, R. K. O.; Rueda, D. S. Optimal molecular crowding accelerates group II intron folding and maximizes catalysis. Proc. Natl. Acad. Sci. U.S.A. 2018, 115, 11917.

(11) Pastor, I.; Vilaseca, E.; Madurga, S.; Garcés, J. L.; Cascante, M.; Mas, F. Effect of Crowding by Dextrans on the Hydrolysis of NSuccinyl-1-phenyl-Ala-p-nitroanilide Catalyzed by $\alpha$-Chymotrypsin. J. Phys. Chem. B 2011, 115, 1115-1121.

(12) Martin, J. Requirement for GroEL/GroES-Dependent Protein Folding under Nonpermissive Conditions of Macromolecular Crowding. Biochemistry 2002, 41, 5050-5055.

(13) Ferreira, L. A.; Uversky, V. N.; Zaslavsky, B. Y. Role of solvent properties of water in crowding effects induced by macromolecular agents and osmolytes. Mol. BioSyst. 2017, 13, 2551-2563.

(14) Alberti, S. Phase separation in biology. Curr. Biol. 2017, 27, R1097-R1102. 
(15) Tian, L.; Martin, N.; Bassindale, P. G.; Patil, A. J.; Li, M.; Barnes, A.; Drinkwater, B. W.; Mann, S. Spontaneous assembly of chemically encoded two-dimensional coacervate droplet arrays by acoustic wave patterning. Nat. Commun. 2016, 7, 13068.

(16) Brangwynne, C. P.; Eckmann, C. R.; Courson, D. S.; Rybarska, A.; Hoege, C.; Gharakhani, J.; Julicher, F.; Hyman, A. A. Germline P Granules Are Liquid Droplets That Localize by Controlled Dissolution/Condensation. Science 2009, 324, 1729.

(17) Feric, M.; Vaidya, N.; Harmon, T. S.; Mitrea, D. M.; Zhu, L.; Richardson, T. M.; Kriwacki, R. W.; Pappu, R. V.; Brangwynne, C. P. Coexisting Liquid Phases Underlie Nucleolar Subcompartments. Cell 2016, 165, 1686-1697.

(18) Brangwynne, C. P.; Mitchison, T. J.; Hyman, A. A. Active liquid-like behavior of nucleoli determines their size and shape in Xenopus laevis oocytes. Proc. Natl. Acad. Sci. U.S.A. 2011, 108, 4334.

(19) Patel, A.; Lee, H. O.; Jawerth, L.; Maharana, S.; Jahnel, M.; Hein, M. Y.; Stoynov, S.; Mahamid, J.; Saha, S.; Franzmann, T. M.; Pozniakovski, A.; Poser, I.; Maghelli, N.; Royer, L. A.; Weigert, M.; Myers, E. W.; Grill, S.; Drechsel, D.; Hyman, A. A.; Alberti, S. A Liquid-to-Solid Phase Transition of the ALS Protein FUS Accelerated by Disease Mutation. Cell 2015, 162, 1066-1077.

(20) Albertsson, P.-Å.; Sasakawa, S.; Walter, H. Cross Partition and Isoelectric Points of Proteins. Nature 1970, 228, 1329-1330.

(21) Diamond, A. D.; Hsu, J. T. Protein partitioning in PEG/dextran aqueous two-phase systems. AIChE J. 1990, 36, 1017-1024.

(22) Diamond, A. D.; Hsu, J. T. Fundamental studies of biomolecule partitioning in aqueous two-phase systems. Biotechnol. Bioeng. 1989, 34, 1000-1014.

(23) Grilo, A. L.; Raquel Aires-Barros, M.; Azevedo, A. M. Partitioning in Aqueous Two-Phase Systems: Fundamentals, Applications and Trends. Sep. Purif. Rev. 2016, 45, 68-80.

(24) Asenjo, J. A.; Andrews, B. A. Aqueous two-phase systems for protein separation: Phase separation and applications. J. Chromatogr. A 2012, 1238, 1-10.

(25) Asenjo, J. A.; Andrews, B. A. Aqueous two-phase systems for protein separation: A perspective. J. Chromatogr. A 2011, 1218, $8826-8835$.

(26) Akbulut, O.; Mace, C. R.; Martinez, R. V.; Kumar, A. A.; Nie, Z.; Patton, M. R.; Whitesides, G. M. Separation of Nanoparticles in Aqueous Multiphase Systems through Centrifugation. Nano Lett. 2012, 12, 4060-4064.

(27) Iqbal, M.; Tao, Y.; Xie, S.; Zhu, Y.; Chen, D.; Wang, X.; Huang, L.; Peng, D.; Sattar, A.; Shabbir, M. A. B.; Hussain, H. I.; Ahmed, S.; Yuan, Z. Aqueous two-phase system (ATPS): an overview and advances in its applications. Biol. Proced. Online 2016, 18, 18.

(28) Zhang, J.; Hwang, J.; Antonietti, M.; Schmidt, B. V. K. J. Waterin-Water Pickering Emulsion Stabilized by Polydopamine Particles and Cross-Linking. Biomacromolecules 2019, 20, 204-211.

(29) Zhang, J.; Kumru, B.; Schmidt, B. V. K. J. Supramolecular Compartmentalized Hydrogels via Polydopamine Particle-Stabilized Water-in-Water Emulsions. Langmuir 2019, 35, 11141-11149.

(30) Ganley, W. J.; Ryan, P. T.; van Duijneveldt, J. S. Stabilisation of water-in-water emulsions by montmorillonite platelets. J. Colloid Interface Sci. 2017, 505, 139-147.

(31) Dewey, D. C.; Strulson, C. A.; Cacace, D. N.; Bevilacqua, P. C.; Keating, C. D. Bioreactor droplets from liposome-stabilized allaqueous emulsions. Nat. Commun. 2014, 5, 4670.

(32) Hann, S. D.; Stebe, K. J.; Lee, D. AWE-somes: All Water Emulsion Bodies with Permeable Shells and Selective Compartments. ACS Appl. Mater. Interfaces 2017, 9, 25023-25028.

(33) Pir Cakmak, F.; Keating, C. D. Combining Catalytic Microparticles with Droplets Formed by Phase Coexistence: Adsorption and Activity of Natural Clays at the Aqueous/Aqueous Interface. Sci. Rep. 2017, 7, 3215.

(34) Cacace, D. N.; Rowland, A. T.; Stapleton, J. J.; Dewey, D. C.; Keating, C. D. Aqueous Emulsion Droplets Stabilized by Lipid Vesicles as Microcompartments for Biomimetic Mineralization. Langmuir 2015, 31, 11329-11338.
(35) Keating, C. D. Aqueous Phase Separation as a Possible Route to Compartmentalization of Biological Molecules. Acc. Chem. Res. 2012, $45,2114-2124$.

(36) Dominak, L. M.; Gundermann, E. L.; Keating, C. D. Microcompartmentation in Artificial Cells: pH-Induced Conformational Changes Alter Protein Localization. Langmuir 2010, 26, 56975705.

(37) Long, M. S.; Jones, C. D.; Helfrich, M. R.; Mangeney-Slavin, L. K.; Keating, C. D. Dynamic microcompartmentation in synthetic cells. Proc. Natl. Acad. Sci. U.S.A. 2005, 102, 5920-5925.

(38) Mace, C. R.; Akbulut, O.; Kumar, A. A.; Shapiro, N. D.; Derda, R; Patton, M. R.; Whitesides, G. M. Aqueous Multiphase Systems of Polymers and Surfactants Provide Self-Assembling Step-Gradients in Density. J. Am. Chem. Soc. 2012, 134, 9094-9097.

(39) Davis, B. W.; Aumiller, W. M.; Hashemian, N.; An, S.; Armaou, A.; Keating, C. D. Colocalization and Sequential Enzyme Activity in Aqueous Biphasic Systems: Experiments and Modeling. Biophys. J. 2015, 109, 2182-2194.

(40) Pozdnyakova, I.; Wittung-Stafshede, P. Non-linear effects of macromolecular crowding on enzymatic activity of multi-copper oxidase. Biochim. Biophys. Acta, Proteins Proteomics 2010, 1804, 740744.

(41) Bradford, M. M. Rapid and sensitive method for quantitation of microgram quantities of protein utilizing principle of protein-dye binding. Anal. Biochem. 1976, 72, 248-254.

(42) Zor, T.; Selinger, Z. Linearization of the bradford protein assay increases its sensitivity: Theoretical and experimental studies. Anal. Biochem. 1996, 236, 302-308.

(43) Johnson, K. A.; Goody, R. S. The original Michaelis constant: translation of the 1913 Michaelis-Menten paper. Biochemistry 2011, 50, 8264-8269.

(44) Miranda, M. V.; Lahore, H. m. F.; Cascone, O. Horseradish peroxidase extraction and purification by aqueous two-phase partition. Appl. Biochem. Biotechnol. 1995, 53, 147-154.

(45) Forciniti, D.; Hall, C. K.; Kula, M. R. Interfacial tension of polyethyleneglycol-dextran-water systems: influence of temperature and polymer molecular weight. J. Biotechnol. 1990, 16, 279-296.

(46) Pavlovic, M.; Huber, R.; Adok-Sipiczki, M.; Nardin, C.; Szilagyi, I. Ion specific effects on the stability of layered double hydroxide colloids. Soft Matter 2016, 12, 4024-4033.

(47) Pavlovic, M.; Rouster, P.; Oncsik, T.; Szilagyi, I. Tuning colloidal stability of layered double hydroxides: From monovalent ions to polyelectrolytes. ChemPlusChem 2017, 82, 121-131.

(48) Rouster, P.; Pavlovic, M.; Szilagyi, I. Destabilization of titania nanosheet suspensions by inorganic salts: Hofmeister series and Schulze-Hardy rule. J. Phys. Chem. B 2017, 121, 6749-6758.

(49) Oncsik, T.; Trefalt, G.; Borkovec, M.; Szilagyi, I. Specific Ion Effects on Particle Aggregation Induced by Monovalent Salts within the Hofmeister Series. Langmuir 2015, 31, 3799-3807.

(50) Albertsson, P.-Å. Partition of Cell Particles and Macromolecules in Polymer Two-Phase Systems. In Advances in Protein Chemistry; Anfinsen, C. B., Edsall, J. T., Richards, F. M., Eds.; Academic Press, 1970; Vol. 24, pp 309-341.

(51) Haynes, C. A.; Carson, J.; Blanch, H. W.; Prausnitz, J. M. Electrostatic potentials and protein partitioning in aqueous two-phase systems. AIChE J. 1991, 37, 1401-1409.

(52) Fonin, A.; Silonov, S.; Sitdikova, A.; Kuznetsova, I.; Uversky, V.; Turoverov, K. Structure and Conformational Properties of dGlucose/d-Galactose-Binding Protein in Crowded Milieu. Molecules 2017, 22, 244.

(53) Cussler, E. L. Diffusion: Mass Transfer in Fluid Systems; Cambridge University Press, 1997.

(54) Ando, T.; Skolnick, J. Crowding and hydrodynamic interactions likely dominate in vivo macromolecular motion. Proc. Natl. Acad. Sci. U.S.A. 2010, 107, 18457-18462. 